\title{
6 \\ Collapsing granular beds: The role of interstitial air
}

\author{
Tess Homan, Christa Gjaltema, and Devaraj van der Meer \\ Physics of Fluids Group, Mesa+ Institute for Nanotechnology, and J. M. Burgers Centre for Fluid Dynamics, \\ University of Twente, 7522 NB Enschede, The Netherlands
}

(Received 15 October 2013; published 9 May 2014)

\begin{abstract}
A prefluidized sand bed consisting of fine particles compactifies when it is subjected to a shock. We observe that the response depends on both the shock strength and the ambient pressure, where, counterintuitively, at high ambient pressure the compaction is larger, which we connect to a decrease of the static friction inside the bed. We find that the interstitial air is trapped inside the bed during and long after compaction. We deduce this from measuring the pressure changes above and below the bed: The top pressure decreases abruptly, on the time scale of the compaction, whereas that below the bed slowly rises to a maximum. Subsequently, both gently relax to ambient values. We formulate a one-dimensional diffusion model that uses only the change in bed height and the ambient pressure as an input, and we show that it leads to a fully quantitative understanding of the measured pressure variations.
\end{abstract}

DOI: 10.1103/PhysRevE.89.052204

\section{INTRODUCTION}

The presence of air as an interstitial fluid is known to have a significant influence on the dynamics of a granular medium consisting of small grains (diameter $d<1 \mathrm{~mm}$ ). Well-known examples include the Brazil nut effect, in which air may cause an intruder to rise instead of sink [1,2]; Faraday heaping, where the presence of air leads to the formation of heaps in a vibrated layer of grains [3-6]; inverse Chladni patterns, where air drag pushes small particles on a resonating plate to the antinodes rather than to the nodal lines $[3,7,8]$; the so-called blown air effect, when a cylindrical shell penetrates into a sand bed [9]; and the impact of an object on a prefluidized bed of fine sand, where the presence of air has a profound influence on the splash shape, bed response, and jet formation [10-15].

The understanding of the precise role the air plays is partial at most, due to the complexity of the above experiments together with the infeasibility of measuring the airflow inside the granular medium. We therefore turn to a well-defined experiment in which the role of air can be measured and quantitatively understood, namely the compaction of a homogeneous and loose sand bed after being subjected to a single shock.

Previous compaction research focused on density change as a function of the number of compaction pulses, generally applied by a shaker [16-22]. There the experiments are almost exclusively carried out under vacuum to minimize cohesion forces and to avoid the influence of the interstitial fluid [23]. It was found that compaction decreases with the amount of taps, eventually leading to a static state in which the bed no longer compactifies.

In this work, we study the influence of the air during a single compaction event of an initially loosely packed sand bed. Here the grains have just enough contacts to form a stable configuration, but the slightest shock will destroy this fragile state and compactify the system. If the grains are sufficiently small —as in our experiment - the role played by the air within the pores is crucial. We show that the interstitial air is trapped upon compaction: The pressures above and below the bed are measured during and after the collapse and subsequently connected to a one-dimensional diffusion model to fully understand the behavior of the air inside the sand.
PACS number(s): 45.70.Cc, 47.56.+r

\section{EXPERIMENT}

The common way to compactify a sand bed is by tapping or shaking the bed. We choose to apply a controlled shock using a metal ball connected to a string forming a pendulum, which is attached close to a container filled with sand [see Fig. 1(a)]. By releasing the ball a certain distance $(d)$ from the container wall, the impact strength can be varied.

The container $\left(14 \times 14 \times 100 \mathrm{~cm}^{3}\right)$ is filled up to height $H=40 \mathrm{~cm}$ with sand, which is polydisperse with sizes ranging from 20 to $60 \mu \mathrm{m}$ and a sphericity between 0.2 and 0.6. Pressurized air is blown from below into the container to fluidize the bed. Subsequently, this airflow is very slowly turned off, resulting in a packing fraction $\phi$ of the bed of around 0.41 [24]. Using a vacuum pump, the pressure $P_{0}$ inside the container can be lowered by slowly pumping out air from above and below the bed, such that the packing of the sand bed remains unchanged [11].

The pendulum's mass equals $520 \mathrm{~g}$ and is released from a maximum distance of $d=32 \mathrm{~cm}$. For every $d$, the impact velocity of the ball is measured $(0.14-0.77 \mathrm{~m} / \mathrm{s})$, leading to an impulse imparted on the container between 0.073 and 0.40 $\mathrm{N} \mathrm{s}$. The shock velocity in the sand is of the order of $100 \mathrm{~m} / \mathrm{s}$ [25], such that it would take approximately $1 \mathrm{~ms}$ for the shock to reach the other side of the container. As soon as the ball hits the container wall, the sand bed starts to collapse, and the surface level is monitored during the experiment using a high-speed camera. The bed is observed to collapse as a whole, i.e., the surface of the bed remains flat and the change in bed height is well-defined.

The bed level decreases almost linearly with time and takes a typical time of $30 \mathrm{~ms}$ to reach its final height $H$; see Fig. 2(a). The collapse time is much larger than the time it takes for information to travel through the bed, so we can assume a homogeneous bed collapse. For higher impact speeds, there are small, damped oscillations visible around this final height, possibly triggered by a vibration of the whole container after the impact or a stick-slip motion of the bed during collapse [26]. Larger impact strengths of the ball result, naturally, in a larger drop of the bed and a faster bed height decrease [Figs. 2(c) and 2(a), respectively], indicating a more violent collapse. 


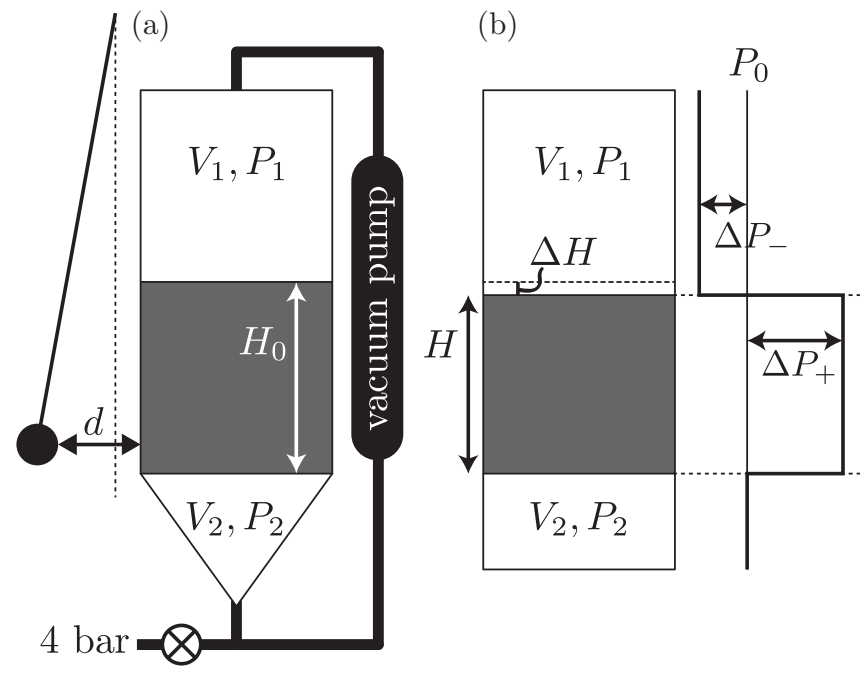

FIG. 1. (a) Schematic view of the experimental setup. A container filled with sand is fluidized from below by pressurized dry air (4 bar). When the airflow is turned off slowly, the sand settles toward a height $H_{0}$. A metal ball hanging from a wire is released at a distance $d$, hitting the container wall and collapsing the fragile sand bed. Meanwhile the pressure above $\left(P_{1}\right)$ and below $\left(P_{2}\right)$ the bed is measured relative to the ambient pressure $P_{0}$, which can be lowered using a vacuum pump. (b) To model the effect of the compaction, as an initial condition the sand bed is collapsed over a distance $\Delta H$ to a height $H$, causing a pressure drop above the bed $\left(\Delta P_{-}\right)$and an increase of the air pressure within it $\left(\Delta P_{+}\right)$. The excess air will relax until there is no remaining pressure difference in the setup.

During and after the impact, the pressure above and below the bed is measured. Both high-speed, differential pressure sensors (SENSIRION SPD600) compare the pressure in the closed-off parts of the container above or below the bed with an external reservoir that is brought to the same initial ambient pressure $\left(P_{0}\right)$ and then sealed.

As soon as the ball hits the container wall, the pressure $\Delta P_{1}$ above the bed decreases sharply, as indicated by any of the light grey (orange) lines in Fig. 3(a). This happens on a time scale of $30 \mathrm{~ms}$, comparable to the time the bed needs to collapse. The pressure $\Delta P_{2}$ below the bed (dark grey (blue) lines) increases, and at a much slower pace. After the initial pressure drop $\left(\Delta P_{1}\right)$ and rise $\left(\Delta P_{2}\right)$, the pressure differences relax back to zero. The different lines in Fig. 3(a) represent measurements done for various impact strengths. The extrema of both $\Delta P_{1}$ and $\Delta P_{2}$ increase linearly with the height change $\Delta H$-and therefore also with $d$-whereas the relaxation of the total pressure difference over the bed is independent of impact speed.

The inset in Fig. 3(a) shows the signal above the bed just after a strong impact $(d=30 \mathrm{~cm})$. There are clear oscillations that are consistent with the top volume oscillation caused by the oscillating bed height.

In the second set of experiments, the impact strength is kept constant $(d=30 \mathrm{~cm})$, but the container is brought to lower ambient pressures. The change in bed height is plotted as a function of ambient pressure in Fig. 2(c) (green squares). Here we find that the bed collapses slightly less for lower (a)

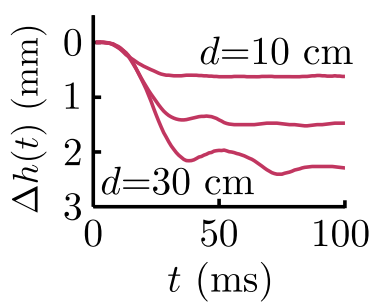

(b)

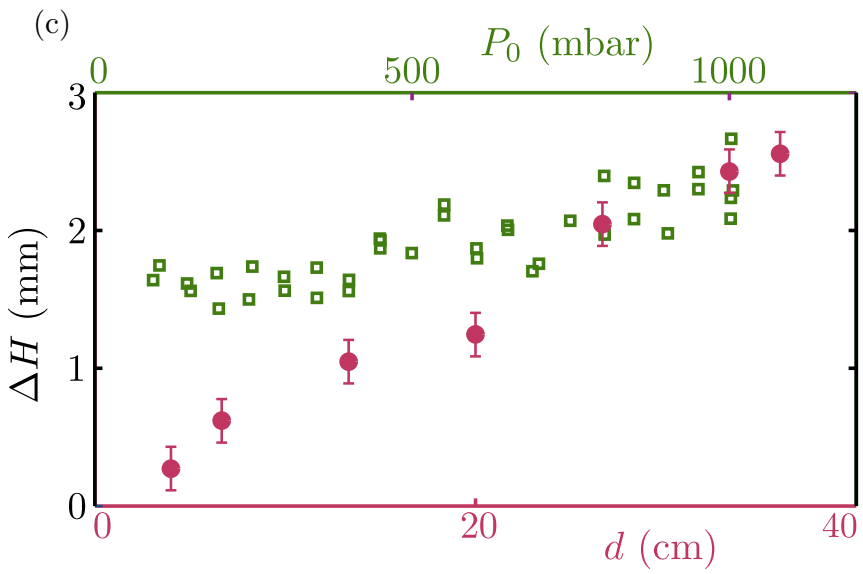

FIG. 2. (Color online) (a) Change in bed height $\Delta h(t)$ as a function of time for three different release distances of the ball: $d=10,20$, and $30 \mathrm{~cm}$. (b) Bed compaction for experiments with the same release distance $(d=30 \mathrm{~cm})$ but at different ambient pressures ( $P_{0}=150,350$, and 950 mbar). (c) The final bed height change $\Delta H$ increases linearly with the release distance $d$ of the impacting object [red (bottom) axis, circles], whereas the bed compaction is slightly less for lower ambient air pressures $P_{0}$ [green (top) axis, open squares].

ambient pressures, while the collapse speed is independent of the pressure [Fig. 2(b)].

The magnitude of the differential pressure signals decreases significantly for lower ambient pressures: The pressure drop above the bed is less, and also the maximum pressure reached below the bed decreases. A big difference with results obtained from varying the shock strength lies in the time constant of the relaxation: In Fig. 3(b), a log-lin plot of the total pressure difference $\Delta P \equiv \Delta P_{2}-\Delta P_{1}$ over the bed for measurements performed at different ambient pressures is shown. Over a fairly large time interval, the signals are linear, indicating an exponential decay. The line bends at the end because the setup is never perfectly airtight, causing a slight increase in the container pressure during the experiment. In addition, the sensors are leaking air into the reservoir due to the measurement method, which also contributes to this effect. The slope decreases when lowering the ambient pressure, indicating a larger relaxation time.

\section{MODEL}

We will now analyze the above results using a model based on Darcy's law, details of the derivation can be found in the Appendix. The starting point is a sand bed of height $H_{0}$ with a certain initial packing fraction $\phi_{0}$. During the very fast collapse of the bed over a distance $\Delta H$ to a new height $H$ and packing 
(a)

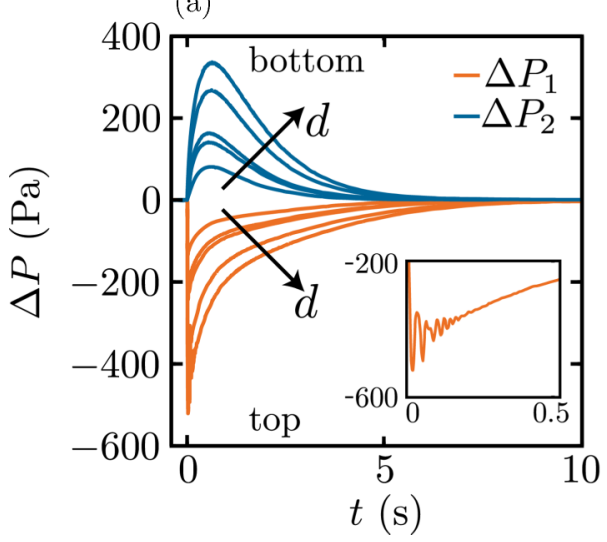

(b)

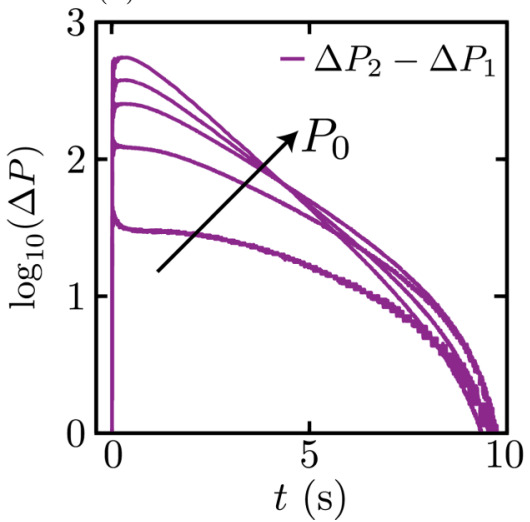

(c)

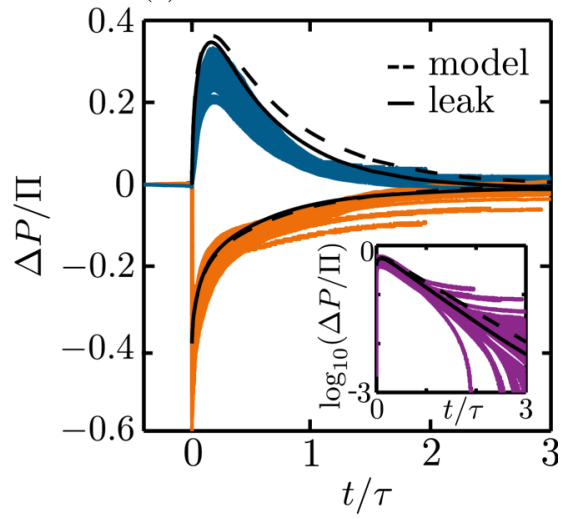

FIG. 3. (Color online) (a) Pressure changes $\Delta P_{1}$ (orange) and $\Delta P_{2}$ (blue) in the container above and below the bed vs time for different values of the release distance $(d=10,15,20,25$, and $30 \mathrm{~cm})$. Inset: When zooming in on $\Delta P_{1}$ for $d=30 \mathrm{~cm}$, we see oscillations upon impact that correlate with those in Fig. 2(a). (b) Logarithm of the pressure difference $\Delta P=\Delta P_{2}-\Delta P_{1}$ over the entire bed for different ambient pressures $\left(P_{0}=200,400,600,800\right.$, and 1000 mbar). (c) Dimensionless pressure change $\Delta P_{1,2} / \Pi$ as a function of dimensionless time $t / \tau$ including all curves in (a) and (b). The data collapse nicely onto a single curve. The dashed and solid black lines represents the dimensionless model without and with corrections for the sensor leak, respectively. The inset shows the logarithm of the dimensionless pressure difference $\left(\Delta P_{2}-\Delta P_{1}\right) / \Pi$ vs $t / \tau$, again in comparison to the model.

fraction $\phi$, air has insufficient time to leave the bed and is therefore trapped within the sand. From the experiments we learn that this is a good assumption, because the bed collapses in typically $30 \mathrm{~ms}$, whereas pressure variations occur in the time scale of seconds. When the bed collapses, the packing fraction of the sand increases, and the pressure of the air within the sand has to rise with an amount $\Delta P_{+}$, since it is confined to a smaller volume. As a consequence, the volume above the sand increases and the pressure drops with $\Delta P_{-}$. Quantitatively, since the interstitial volume decreases from $\left(1-\phi_{0}\right) H_{0} A$ to $(1-\phi) H A$ and the total volume occupied by the sand particles is unchanged $\left(\phi_{0} H_{0} A=\phi H A\right), \Delta P_{+}$ and $\Delta P_{-}$are (to linear order in $\Delta H$ and assuming isothermal compression)

$$
\Delta P_{+}=P_{0} \frac{\Delta H}{(1-\phi) H}, \quad \Delta P_{-}=-P_{0} \frac{A \Delta H}{V_{1}},
$$

where $V_{1}$ is the volume above the collapsed sand bed. In the nonchanging volume below the bed, the pressure remains initially ambient. These initial conditions are illustrated in Fig. 1(b).

The factor that limits the dynamics, i.e., the slow flow of the air inside the sand bed, is governed by Darcy's law, $\vec{q}=$ $-(\kappa / \mu) \vec{\nabla} P$, where $\vec{q}$ is the volume flux, $\kappa \approx 5.5 \times 10^{-12} \mathrm{~m}^{2}$ is the (measured) permeability of the sand bed [27], and $\mu=$ $1.98 \times 10^{-5} \mathrm{~Pa} \mathrm{~s}$ is the dynamic viscosity of air. Combining Darcy's law with the ideal gas law and linearizing around $P_{0}$ leads to a diffusion equation for the pressure rise $\Delta P_{S}$ inside the sand,

$$
\frac{\partial \Delta P_{s}}{\partial t}=D \nabla^{2}\left(\Delta P_{s}\right)
$$

where the diffusion coefficient is given by $D=\kappa P_{0} /[\mu(1-$ $\phi)]$. We assume the surface of the bed remains flat during the collapse, and we neglect the effect of the container walls such that the problem can be described by a one-dimensional form of Eq. (2) for $\Delta P_{s}(z, t)$. This equation needs to be supplemented by mass conservation in the top and bottom part of the container (with volume $V_{2}$ ),

$$
\begin{aligned}
& \frac{d \Delta P_{1}}{d t}=-\left.\frac{A}{V_{1}} \frac{\kappa P_{0}}{\mu} \frac{\partial \Delta P_{s}}{\partial z}\right|_{z=H}, \\
& \frac{d \Delta P_{2}}{d t}=\left.\frac{A}{V_{2}} \frac{\kappa P_{0}}{\mu} \frac{\partial \Delta P_{s}}{\partial z}\right|_{z=0},
\end{aligned}
$$

together with the initial conditions from Eq. (1): $\Delta P_{1}(0)=$ $\Delta P_{-}, \Delta P_{s}(z, 0)=\Delta P_{+}$, and $\Delta P_{2}(0)=0$.

This boundary value problem can be nondimensionalized using bed height $\zeta \equiv H$, diffusion time $\tau \equiv H^{2} / D$, and initial pressure in the sand bed $\Pi \equiv \Delta P_{+}$as the typical length, time, and pressure scale, respectively (see the Appendix), leaving us with a problem that contains two parameters only:

$$
\alpha \equiv \frac{(1-\phi) A H}{V_{1}} \approx 0.39, \quad \beta \equiv \frac{V_{1}}{V_{2}} \approx 4.0,
$$

which are the ratio of the interstitial volume within the collapsed sand bed to that above it, and the ratio of the volumes above and below the bed, respectively. Most importantly, since $\Delta H \lesssim 4 \mathrm{~mm}$ is much smaller than $H=H_{0}-\Delta H \approx$ $400 \mathrm{~mm}$, both $\alpha$ and $\beta$ are fixed, such that there is no free parameter in this problem.

First we rescale all experiments, including those of Figs. 3(a) and 3(b), using the pressure and time scales $\tau$ and $\Pi$ [Fig. 3(c)]. Indeed all measurements (for various shock strengths and different ambient pressures) collapse onto a single master curve. The variation largely originates from the difficult measurement of $\Delta H$ where the height decrease of the entire bed has to be estimated from its decrease along one of the side walls. In addition, given that for every experiment the sand bed has to be prepared separately, slight deviations in the final state cause part of the variation. Curves that do not tend to zero but to a constant correspond to experiments at very low ambient pressure, where likely some air was leaking into the container during the measurements. The inset of Fig. 3(c) 
shows that also the decay behavior is neatly collapsed. This is due to the diffusion time $\tau=H^{2} / D=(1-\phi) \mu H^{2} /\left(\kappa P_{0}\right)$ being inversely proportional to the ambient pressure $P_{0}$.

Secondly, we numerically solve the dimensionless boundary value problem corresponding to Eqs. (1)-(3), and we compare it to the experiments. This leads to the black dashed line in Fig. 3(c). Both above and below the bed, the agreement is quite good. In particular, the starting value $\Delta P_{-}$of $\Delta P_{1}$ and the maximum in $\Delta P_{2}$ are nicely predicted. The time scale of the relaxation below the bed, however, seems to be slightly underestimated by the model [Fig. 3(c)]. This can be traced back to the fact that the sensors are leaky [28]. This (known) leak is readily incorporated into the model by adjusting the mass balance of Eqs. (3), leading to a much better agreement [solid line in Fig. 3(c)].

\section{CONCLUSION}

We studied the response of a fine and loose sand bed during a single compaction event. Whereas the visible collapse appears to be concluded within $50 \mathrm{~ms}$, we show that the compaction process continues until all of the excess air manages to escape from the bed, at a time interval exceeding 5 s, i.e., two orders of magnitude larger.

We found that applying a stronger shock compactifies the bed more and faster. The collapse traps air inside the bed and decreases the pressure above it almost instantly, whereafter, on a much larger time scale, the air slowly flows back from and through the bed into the upper container. The pressure below the bed is initially unchanged, but excess pressure of the interstitial air inside the bed causes it to increase. After it reaches a maximum, the pressure will decrease until there is no pressure change left in the bed. The magnitude of the pressure signals in the container increases linearly with the change in bed height caused by stronger shocks.

By varying the ambient air pressure, we obtain a better understanding of the influence of air. Reducing the pressure decreases the measured pressure differences above and below the bed, and it increases the time it takes for the buildup pressure difference to decay. All pressure observations are fully accounted for by a model based on Darcy's flow inside the sand bed.

We observed a small but measurable increase of compaction of the sand bed for increasing ambient air pressures, which is counterintuitive: If pressure-driven forces in the air were counteracting compaction, then they would be increasing with $P_{0}$, and thus they would lead instead to a compaction decrease. So how can the presence of air amplify the compaction process? Part of the answer could lie in air working as a lubrication layer between the particles. Friction between grains eventually stops the grains from falling down and helps it to form a new packing. In a tilt table experiment, we have measured, after the fluidization procedure, the angle of repose $\theta$ at which the sand started to flow at atmospheric and reduced pressure, giving $\theta=15.8 \pm 0.9^{\circ}$ at 1 bar and $\theta=18.5 \pm 0.8^{\circ}$ at 100 mbar. For low ambient pressure, the grains start to flow at a higher angle, indicating a larger friction between the sand grains when there is very little air. This is in agreement with the larger collapse for high ambient pressures: If air reduces the friction between the grains, they will be slowed down less and therefore the bed will be more compactified when the grains have found their final position. The precise mechanism by which this happens remains to be elucidated.

\section{ACKNOWLEDGMENTS}

This work is part of the research programme of the Foundation for Fundamental Research on Matter (FOM), which is part of the Netherlands Organisation for Scientific Research (NWO).

\section{APPENDIX: DERIVATION OF THE MODEL}

In this Appendix, we derive the diffusion model based on Darcy's law for air flow in a collapsed sand bed that has been used in the main text of the paper. In doing so, we assume that the compaction of the bed will have occurred uniformly, leading to a uniform compression of the air within the pores between the sand grains, and that as a result flow will take place in the vertical $(z)$ direction only.

\section{Linearized porous media equation}

For an infinitesimally small cube of sand of volume $(d V=$ $d x d y d z$ ), the mass balance equation for a one-dimensional flow in the $z$ direction reads

$$
\frac{d m}{d t}=\rho Q_{z}-\rho Q_{z+d z}
$$

where $\rho Q_{z}$ and $\rho Q_{z+d z}$ give the in- and outflow of mass at the bottom and the top of the volume. Here, the air density $\rho$ may vary with pressure. The in- and outflow can be calculated using Darcy's law, which describes the volume flow rate through a porous medium,

$$
\vec{Q}=-A \frac{\kappa}{\mu} \vec{\nabla} P_{s},
$$

where $A$ is the area perpendicular to the flow (in this case $d x d y$ ), $\kappa$ is the permeability of the porous material, $\mu$ is the viscosity of the flowing medium (in our case air), and $P_{s}$ is the pressure of the air in the control volume. Inserting Darcy's law into Eq. (A1) and expanding around $z$ leads to

$$
\frac{d m}{d t}=\frac{\kappa}{\mu} \frac{\partial}{\partial z}\left[\rho \frac{\partial P_{s}}{\partial z}\right] d V .
$$

The volume of air in the cube depends on the packing fraction $\phi$ of the porous material. If we assume that the packing fraction is not changing, we can rewrite the left-hand side of Eq. (A3) as

$$
\frac{d m}{d t}=(1-\phi) \frac{\partial \rho}{\partial t} d V
$$

From the ideal gas law, we know that $\rho=P_{s} /(R T)$, such that we can replace the density in Eqs. (A3) and (A4) with $P_{s}$. Assuming that the temperature $T$ is constant, due to the good thermal contact of the gas with the porous media, which has a relatively large heat capacity, this then leads to

$$
\frac{\partial P_{s}}{\partial t}=\frac{\kappa}{2 \mu(1-\phi)} \frac{\partial^{2} P_{s}^{2}}{\partial z^{2}},
$$


which is the well-known porous media equation in the limit we are discussing now. Writing $P_{s}$ as a constant ambient pressure plus a small pressure difference $\left(P_{s}=P_{0}+\Delta P_{s}\right)$ and linearizing around $P_{0}$ leads to a diffusion equation for flow in a porous material,

$$
\frac{\partial \Delta P_{s}}{\partial t}=\frac{\kappa P_{0}}{\mu(1-\phi)} \frac{\partial^{2} \Delta P_{s}}{\partial z^{2}},
$$

with diffusivity $D \equiv \kappa P_{0} /[\mu(1-\phi)]$. Note that $\Delta P_{s} / P_{0} \approx$ 0.005 for our experiments, which justifies neglecting the nonlinear terms in Eq. (A5).

\section{Initial conditions after bed collapse}

The diffusion equation describes the dynamics of the air in the sand in the presence of pressure gradients. For the current problem, these gradients arise from the collapse of the bed, as a result of which the pressure above the bed decreases and the pressure inside the bed increases. The volume occupied by the sand grains is constant, which, if we assume the bed compactifies homogeneously, relates the packing fraction before $\left(\phi_{0}\right)$ and after the collapse $(\phi)$ as

$$
\phi=\phi_{0} \frac{H_{0}}{H}=\phi_{0} \frac{H+\Delta H}{H},
$$

where $H_{0}$ and $H=H_{0}-\Delta H$ are the bed height before and after collapse, respectively. The interstitial volume changes from $\left(1-\phi_{0}\right) A H_{0}$ before to $(1-\phi) A H$ after collapse, with $A$ the cross-sectional area of the container. Now, assuming that the collapse of the bed is so fast that air has no time to escape, the (isothermal) ideal gas law has to be satisfied before and after the collapse:

$$
\left(1-\phi_{0}\right) A H_{0} P_{0}=(1-\phi) A H\left(P_{0}+\Delta P_{+}\right) .
$$

Combining relations (A7) and (A8) leads to an equation for the initial pressure rise $\Delta P_{+}$inside the bed:

$$
\Delta P_{+}=\frac{P_{0} \Delta H}{(1-\phi) H} .
$$

Simultaneously, the (initially ambient) pressure $P_{1}=P_{0}$ in the container volume $V_{1}$ above the bed will decrease by an amount $\Delta P_{-}$due to an increase in the volume: $P_{0} V_{1}=$ $\left(P_{0}+\Delta P_{-}\right)\left(V_{1}+A \Delta H\right)$. This leads to

$$
\Delta P_{-} \approx-\frac{A \Delta H}{V_{1}} P_{0}
$$

where we neglect the higher-order terms in $A \Delta H / V_{1}$ because the volume of the top part of the container is of the order of the total volume of the sand bed $\left(V_{1} \sim A H\right)$ such that $A \Delta H / V_{1} \approx \Delta H / H \approx 0.01$. Finally, the pressure below the bed will not change during the collapse. The initial conditions are illustrated in the right half of Fig. 1(b).

\section{Boundary conditions}

Now that we have introduced a pressure difference in the setup, the pressure will be equalized by air moving from the sand bed into the top and bottom part of the container. Using Darcy's law [Eq. (A2)], the time rate of change of the amount of air $m_{1}$ in the volume $V_{1}$ above the bed is proportional with the inflow from the bed,

$$
\frac{d m_{1}}{d t}=\rho_{1} Q=-\left.\rho_{1} A \frac{\kappa}{\mu} \frac{\partial P_{s}}{\partial z}\right|_{z=H},
$$

and proportional to the time rate of change of the density $\rho_{1}$ given that the volume $\left(V_{1}\right)$ remains constant,

$$
\frac{d m_{1}}{d t}=\frac{\partial}{\partial t}\left(\rho_{1} V_{1}\right)=V_{1} \frac{d \rho_{1}}{d t} .
$$

Combining Eqs. (A11) and (A12) with the isothermal ideal gas law $\rho_{1}=P_{1} / R T$, writing $P_{1}=P_{0}+\Delta P_{1}$, and linearizing around $P_{0}$ leads to the boundary condition at the top of the sand bed,

$$
\frac{d \Delta P_{1}}{d t}=-\left.\frac{A}{V_{1}} \frac{\kappa P_{0}}{\mu} \frac{\partial \Delta P_{s}}{\partial z}\right|_{z=H} .
$$

For the container volume $V_{2}$ below the sand bed, we find similarly that

$$
\frac{d \Delta P_{2}}{d t}=\left.\frac{A}{V_{2}} \frac{\kappa P_{0}}{\mu} \frac{\partial \Delta P_{s}}{\partial z}\right|_{z=0} .
$$

\section{Nondimensionalization}

To nondimensionalize the preceding set of equations, we define a typical length, time, and pressure scale as

$$
\begin{aligned}
& \zeta=H, \quad \tau=\frac{H^{2}}{D}=\frac{\mu(1-\phi) H^{2}}{\kappa P_{0}}, \\
& \Pi=\Delta P_{+}=\frac{P_{0} \Delta H}{(1-\phi) H},
\end{aligned}
$$

such that we can rewrite the boundary value problem consisting of Eqs. (A6), (A9), (A10), (A13), and (A14) in terms of the nondimensional variables $\widetilde{z}=z / \zeta, \widetilde{t}=t / \tau$, and $\Delta \widetilde{P}=\Delta P / \Pi$. This leads to the following partial differential equation:

$$
\frac{\partial \Delta \widetilde{P}_{s}}{\partial \widetilde{t}}=\frac{\partial^{2} \Delta \widetilde{P}_{s}}{\partial \widetilde{z}^{2}},
$$

together with the initial conditions

$$
\Delta \widetilde{P}_{s}(\widetilde{z}, 0)=1, \quad \Delta \widetilde{P}_{1}(0) \approx-\alpha, \quad \Delta \widetilde{P}_{2}(0)=0,
$$

and the boundary equations

$$
\frac{d \Delta \widetilde{P}_{1}}{d \widetilde{t}}=-\left.\alpha \frac{\partial \Delta \widetilde{P}_{s}}{\partial \widetilde{z}}\right|_{\tilde{z}=1}, \quad \frac{d \Delta \widetilde{P}_{2}}{d \widetilde{t}}=\left.\alpha \beta \frac{\partial \Delta \widetilde{P}_{s}}{\partial \widetilde{z}}\right|_{\tilde{z}=0} .
$$

This set of equations contains two parameters only, $\alpha$ and $\beta$, which are the ratio of the free volume within the sand to $V_{1}$ and the ratio of the volumes above and below the bed, respectively,

$$
\alpha=\frac{(1-\phi) A H}{V_{1}}, \quad \beta=\frac{V_{1}}{V_{2}} .
$$

From the dimensions of the setup, both are readily computed. Using the measured values $V_{1}=1.22 \times 10^{-2} \mathrm{~m}^{3}, V_{2}=$ $3.05 \times 10^{-3} \mathrm{~m}^{3}, A=2.03 \times 10^{-2} \mathrm{~m}^{2}, H=0.40 \mathrm{~m}$, and $\phi=$ 0.42 , we find $\alpha=0.39$ and $\beta=4.0$. This fixes the (numerical) solution of the boundary value problem completely. 


\section{Large-time limit}

Long after the collapse, the trapped interstitial air will have largely flown out of the sand bed, such that the pressure gradient $\partial \Delta P_{s} / \partial z$ inside the sand approaches a constant value, i.e., $\left(\Delta P_{1}-\Delta P_{2}\right) / H$, such that

$$
\frac{\partial \Delta \widetilde{P}_{s}}{\partial \widetilde{z}} \approx \Delta \widetilde{P}_{1}-\Delta \widetilde{P}_{2} .
$$

Inserting the above approximation into Eqs. (A18) and subtracting the second expression from the first leads to

$$
\frac{\partial\left(\Delta \widetilde{P}_{1}-\Delta \widetilde{P}_{2}\right)}{\partial \widetilde{t}} \approx-\alpha(\beta+1)\left(\Delta \widetilde{P}_{1}-\Delta \widetilde{P}_{2}\right),
$$

which is readily solved as

$$
\Delta \widetilde{P}_{1}-\Delta \widetilde{P}_{2} \propto \exp [-\alpha(\beta+1) \widetilde{t}] .
$$

The exponential decay of $\Delta \widetilde{P}_{1}-\Delta \widetilde{P}_{2}$ in the large-time limit predicted by Eq. (A22) is clearly observable as the linear behavior in the inset of Fig. 3(c).

\section{Corrections}

The effect of the porous plate can be incorporated into the equations with the introduction of three additional dimensionless parameters, namely the dimensionless thickness of the plate $\widetilde{h}_{p}=h_{p} / H$, the ratio $\gamma$ of the permeabilities of the sand and plate $\left(\gamma=\kappa / \kappa_{p}\right)$, and the ratio $\delta$ of the porosities of the sand and plate $\left[\delta=(1-\phi) /\left(1-\phi_{p}\right)\right]$. For realistic values of the parameters, however, this leads to minor corrections in the numerical solution to the boundary value problem.

A more significant correction stems from the fact that the (SENSIRION SPD600) pressure sensors that were used are leaky due to the measurement method on which they are based, which uses a flow rate measurement to obtain the pressure drop over a capillary. According to the manufacturer, the sensors leak $150 \mathrm{~mL} / \mathrm{min}\left[=2.5 \times 10^{-6} \mathrm{~m}^{3} / \mathrm{s}\right.$ at full scale $( \pm 500$ $\mathrm{Pa})$, which leads to a volume flow rate $Q_{\text {leak }}=-\sigma \Delta P_{i}$ with $\sigma=5.0 \times 10^{-9} \mathrm{~m}^{3} / \mathrm{Pa} \mathrm{s}$ for both the top $(i=1)$ and bottom $(i=2)$ part of the container. This leads to a modification of Eq. (A11): $d m / d t=\rho Q+\rho Q_{\text {leak }}$, the incorporation of which finally amounts to the addition of a term to both boundary conditions (A13) and (A14) which in dimensionless form reads

$$
\begin{gathered}
\frac{d \Delta \widetilde{P}_{1}}{d \widetilde{t}}=-\left.\alpha \frac{\partial \Delta \widetilde{P}_{s}}{\partial \widetilde{z}}\right|_{\tilde{z}=1}-\lambda \Delta \widetilde{P}_{1}, \\
\frac{d \Delta \widetilde{P}_{2}}{d \widetilde{t}}=\left.\alpha \beta \frac{\partial \Delta \widetilde{P}_{s}}{\partial \widetilde{z}}\right|_{\tilde{z}=0}-\lambda \beta \Delta \widetilde{P}_{2},
\end{gathered}
$$

with

$$
\lambda=\frac{\tau P_{0}}{V_{1}} \sigma=\frac{\mu(1-\phi) H^{2}}{\kappa V_{1}} \sigma
$$

Again, $\lambda$ is not a free parameter but is completely fixed by measurable properties of the experiment. Using $\kappa \approx$ $5.5 \times 10^{-12} \mathrm{~m}^{2}, \mu=1.98 \times 10^{-5} \mathrm{~Pa} \mathrm{~s}, H=0.40 \mathrm{~m}, V_{1}=$ $1.22 \times 10^{-2} \mathrm{~m}^{3}$, and $\phi=0.42$, we find $\lambda=0.14$. Note that the magnitude of the correction term in Eq. (A23) is of order $\lambda / \alpha \approx 0.3$ and therefore much larger than the effects of the linearization of the porous media equation and the neglecting of higher-order terms in $\Delta H / H$.
[1] X. Yan, Q. Shi, M. Hou, K. Lu, and C. K. Chan, Phys. Rev. Lett. 91, 014302 (2003).

[2] M. E. Möbius, X. Cheng, G. S. Karczmar, S. R. Nagel, and H. M. Jaeger, Phys. Rev. Lett. 93, 198001 (2004).

[3] M. Faraday, Philos. Trans. R. Soc. London 52, 299 (1831).

[4] H. K. Pak, E. Van Doorn, and R. P. Behringer, Phys. Rev. Lett. 74, 4643 (1995).

[5] T. Shinbrot and F. J. Muzzio, Phys. Rev. Lett. 81, 4365 (1998).

[6] H. J. van Gerner, M. A. van der Hoef, D. van der Meer, and K. van der Weele, Phys. Rev. E 76, 051305 (2007).

[7] E. Chladni, Die Akustik (Breitkopf \& Haertel, Leipzig, 1802).

[8] H. J. van Gerner, K. van der Weele, M. A. van der Hoef, and D. van der Meer, J. Fluid Mech. 689, 203 (2011).

[9] R. Clément, S. C. du Pont, M. Ould-Hamouda, D. Duveau, and S. Douady, Phys. Rev. Lett. 106, 098001 (2011).

[10] J. R. Royer et al., Nat. Phys. 1, 164 (2005).

[11] G. Caballero, R. Bergmann, D. van der Meer, A. Prosperetti, and D. Lohse, Phys. Rev. Lett. 99, 018001 (2007).

[12] J. R. Royer, E. I. Corwin, P. J. Eng, and H. M. Jaeger, Phys. Rev. Lett. 99, 038003 (2007).

[13] J. R. Royer, E. I. Corwin, B. Conyers, A. Flior, M. L. Rivers, P. J. Eng, and H. M. Jaeger, Phys. Rev. E 78, 011305 (2008).

[14] J. R. Royer, B. Conyers, E. I. Corwin, P. J. Eng, and H. M. Jaeger, Europhys. Lett. 93, 28008 (2011).
[15] G. A. Caballero-Robledo et al., Granular Matter 14, 179 (2012).

[16] J. B. Knight, C. G. Fandrich, C. N. Lau, H. M. Jaeger, and S. R. Nagel, Phys. Rev. E 51, 3957 (1995).

[17] E. Nowak et al., Powder Technol. 94, 79 (1997).

[18] T. Boutreux and P. de Gennes, Physica A 244, 59 (1997).

[19] E. Ben-Naim et al., Physica D 123, 380 (1998).

[20] S. J. Linz and A. Dohle, Phys. Rev. E 60, 5737 (1999).

[21] P. Richard et al., Nat. Mater. 4, 121 (2005).

[22] P. Ribiere et al., J. Phys. Condens. Matter 17, S2743 (2005).

[23] In the work of Ribière [22], an open container is used and air might play a role; however, they do not go into details about the influence of air.

[24] The packing fraction is defined as the fraction of space that is occupied by the solid phase, i.e., the sand grains.

[25] C. H. Liu and S. R. Nagel, Phys. Rev. B 48, 15646 (1993).

[26] In both cases, the role of air will be crucial: During collapse, air in the bed is pressurized and therefore (in principle) capable of performing the work necessary to temporarily and slightly reverse the collapse, as observed in the oscillation.

[27] The bed permeability $(\kappa)$ could be estimated using the CarmanKozeny relation, but it was also obtained directly in the current experimental setup by measuring the pressure drop 
over the bed while a constant flow rate was applied through it.

[28] According to the manufacturer, the sensors leak $150 \mathrm{~mL} / \mathrm{min}$ at full scale (500 Pa) due to the measurement method, which uses a flow rate measurement to obtain the pressure drop over a capillary. This method is faster and more accurate than others, but it introduces a small leak. The incorporation of this leak into the model is discussed in the Appendix. 